\title{
Development and Characterization of Molecular Markers Associated with Female Plants in Muscadine Grape
}

\author{
Patrick Conner ${ }^{1}$, Joann Conner, and Paige Catotti \\ Department of Horticulture, University of Georgia-Tifton Campus, 4604 Research Way, Tifton, GA \\ 31793
}

Jennifer Lewter and John R. Clark

Department of Horticulture, University of Arkansas, 316 Plant Sciences Building, Fayetteville, AR 72701

Luiz A. Biasi

Departamento de Fitotecnia e Fitossanitarismo, Universidade Federal do Paraná, Rua dos

Funcionários, 1540, Curitiba, PR, Brazil 80035-050

AdDITIONAL INDEX words. Muscadinia rotundifolia, Vitis rotundifolia, anther, self-fertile, pistillate

\begin{abstract}
Muscadine (Vitis rotundifolia Michx.) vines may be male (M), female (F), or hermaphroditic (H). Male flowers have only filaments and anthers, whereas female and hermaphroditic flowers are morphologically perfect. Female flowers are distinguished from hermaphroditic flowers by their reflexed stamens (as opposed to upright) and nonfunctional pollen. Primers derived from previously identified candidate genes located at the sex locus of Vitis vinifera $\mathrm{L}$. were used to generate amplicons from $\mathrm{M}, \mathrm{F}$, and $\mathrm{H}$ muscadine cultivars. Sequence analysis of the amplicons revealed insertion/deletion (indel) polymorphisms in a trehalose-6-phosphate phosphatase (TPP) gene (VR006) and a WRKY transcription factor 21 gene (VR009). Primers were designed to create diagnostic markers for each indel polymorphism. Associations between the marker alleles and the plant sex trait were examined in a wide range of muscadine germplasm and in segregating populations derived from $\mathrm{F} \times \mathrm{H}, \mathrm{F} \times \mathrm{M}$, and $\mathrm{H} \times \mathrm{H}$ crosses. VR006 produced a codominant marker that was able to differentiate the female-associated allele from the male- and hermaphroditicassociated alleles. The marker was able to detect female plants and will be suitable for screening breeding program progenies. VR009 was able to detect the presence of the female allele in most germplasm, but a crossover event appears to have separated the marker from the sex locus in some germplasm. As shown in previous muscadine genetic studies, $\mathrm{H} \times \mathrm{H}$-derived populations produced male seedlings. Marker analysis of these populations indicates that male flowers only occur in seedlings which are heterozygous for $\mathbf{F}$ - and $\mathbf{H}$-associated marker alleles and inheritance of flower type in muscadine remains unclear.
\end{abstract}

The genus Vitis L. contains two subgenera, Euvitis Planch. (bunch grapes) and Muscadinia Planch. (muscadine grapes). The Muscadinia consists of just three species: $V$. rotundifolia, the common muscadine grape known throughout the southeastern United States, V. munsoniana (Simpson ex Munson) M.O. Moore a semitropical variant of $V$. rotundifolia native to southern Florida, and $V$. popenoei J.H. Fennel a tropical native to southern Mexico. The muscadine grape is the only commonly cultivated member of the Muscadinia subgenus. Muscadine is an important native North American grape species planted mainly in the southeastern United States. The muscadine grape differs from the familiar bunch grape (Vitis labrusca L. and $V$. vinifera) by the presence of smaller clusters, unbranched tendrils, and berries with thick skins and a unique fruity aroma. Muscadine berries often abscise from the cluster when ripe and are harvested as single berries. In addition, the Muscadinia species have 40 chromosomes $(2 \times=2 n=$ $40)$, whereas Euvitis have $38(2 \times=2 \mathrm{n}=38)$.

Received for publication 20 Dec. 2016. Accepted for publication 14 Feb. 2017. We thank Terry Bland with North Carolina State University for obtaining leaf samples from North Carolina for analysis.

${ }^{1}$ Corresponding author. E-mail: pconner@uga.edu.
Native muscadines are typically dioecious (Reimer and Detjen, 1910), although a few naturally occurring hermaphrodite vines have been described (Detjen, 1917). The perfect hermaphroditic muscadine flower, hereafter referred to as hermaphroditic, consists of a normal pistil surrounded by five or more tall, erect stamens producing functional pollen. The filaments equal or exceed the combined length of the ovary, style, and stigma (Detjen, 1917). In contrast, female flowers are imperfect hermaphrodites, hereafter referred to as female, in which the ovary, style, and stigma are thickened and enlarged, and stamens are both recurved and shorter, and the pollen produced is sterile (Detjen, 1917). The staminate flower has merely a whorl of erect stamens containing pollen (Dearing, 1917). It has been proposed that hermaphroditic vines are staminate vines which have regained the ability to produce a functional pistil (Detjen, 1917). Evidence for this theory comes from the report of a vine which bears blossoms of all stages of gradation from true staminate to true hermaphrodite (Dearing, 1917). In addition, hermaphroditic and staminate flower clusters are much larger than female clusters (Detjen, 1917).

Before the 1940s, all fruiting muscadines were female and vineyards typically contained female vines with interspersed male pollenizers. Early on, breeders discovered hermaphroditic 
vines, which were occasionally produced from crosses between male and female vines, and the resulting hermaphrodites were used to develop hermaphroditic cultivars with improved fruit quality (Dearing, 1917). The lineage of all present-day hermaphrodites can be traced back to two original hermaphroditic seedlings known as $\mathrm{H} 1$ and H2 (Dearing, 1948). H1 resulted from a cross between 'Eden' and a $V$. munsoniana cultivar Mission Male, whereas $\mathrm{H} 2$ resulted from a cross between Scuppernong and another $V$. rotundifolia cultivar New Smyrna. Both hermaphrodites were spontaneously formed and were identified from a group of male and female seedlings by flower morphology. A third hermaphroditic selection, 'Hope', was identified from the wild and used in genetic experiments (Detjen, 1917), but its progeny were not used to breed modern hermaphroditic cultivars. As hermaphrodites with acceptable fruit quality were developed, the male pollenizer vines were replaced with hermaphroditic pollenizers in vineyards leading to increased production (Dearing, 1948).

Although $\mathrm{H} 1$ and $\mathrm{H} 2$ are both hermaphroditic, there are differences between them. Genetic studies (Loomis et al., 1954) showed that when self-pollinated, hermaphrodites fell into two groups: those producing hermaphroditic and female progeny and those producing hermaphroditic, female, and male progeny. The first group, which had the H1 source of hermaphroditism, produced three hermaphrodites for every one female. The second group, which had the H2 source of hermaphroditism, produced a ratio of nine hermaphrodites to three females to four males.

Like wild muscadines, wild Euvitis plants are dioecious. However, the domestication of $V$. vinifera ssp. vinifera resulted in the introduction and selection of perfect flowers from an ancient and unknown origin so that most modern cultivars are hermaphroditic. Oberle (1938) proposed sex expression in Vitis is controlled by two linked genes with the dominant alleles, So (suppressions of ovules) and Sp (development of pollen), linked in cis-arrangement. Thus, males would be SoSp/sosp and females would be sosp/sosp. Interestingly, a rare crossover event would produce an soSp/sosp plant that would produce both functional pollen and functional pistils, a possible explanation for the development of hermaphrodites. A more general model proposed by Levadoux (1946) and supported by Carbonneau (1983) and Antcliff (1980) proposes a single major locus with three different alleles: $\mathrm{M}, \mathrm{H}$, and $\mathrm{F}$, in the dominance relationship $\mathrm{M}>\mathrm{H}>\mathrm{F}$, control Vitis sex expression. It should be noted, however, that Carbonneau (1983) found some male plants in crosses between hermaphrodites, leading him to propose that the multiallelic locus is modified by the epistatic action of a gene located 30 map units away.

Several different studies have mapped the sex-determining locus in Euvitis to a single region on Chromosome 2 (Battilana et al., 2013; Dalbó et al., 2000; Marguerit et al., 2009; Riaz et al., 2006). Fechter et al. (2012) used the reference genome sequence for $V$. vinifera to develop additional simple sequence repeat (SSR) markers and subsequent primers for the region around the sex locus in Euvitis. By performing genotyping, screening of a bacterial artificial chromosome (BAC) library, and sequencing BAC clones, they were able to fine map the sex locus. Through this process they identified a candidate gene for flower sex, adenine phosphoribosyltransferase (APT). APT genes are known to metabolize the plant hormone cytokinin, which may influence flower development. Using the APT sequence, they developed polymerase chain reaction (PCR) primers around a large polymorphic 130-bp indel in the second intron to create a marker for the presence or absence of the female alleles. Building on this work, Picq et al. (2014) developed primer pairs to amplify stretches of the promoter and beginning coding regions of 11 genes located near the sex locus, including the APT gene. These amplicons ranged in size from 673 to $1515 \mathrm{bp}$. After sequencing these amplicons in a diverse set of male and female wild grapevines, they found 46 polymorphisms in four amplicons that were perfectly or strongly linked to the sex trait. To determine the origin of the allele for hermaphroditism, they built a network based on the sex macrohaplotypes and the information from the four sexlinked amplicons. This region displayed haplotype diversity, linkage disequilibrium, and differentiation that corresponded to a small XY sex-determining region with XY males and XX females. Diversity and network analysis indicated that hermaphroditic alleles were more closely related to male alleles than to female alleles (Picq et al., 2014).

Flower type expression in muscadine phenotypically and genetically shares many similarities with that of Euvitis. Our objective was to use the primers developed by Picq et al. (2014) to find sequence polymorphisms associated with plant sex in muscadine, and to use these polymorphisms to develop genetic markers for the sex trait in muscadine. Muscadine breeding programs make use of $\mathrm{F} \times \mathrm{H}$ and $\mathrm{H} \times \mathrm{H}$ crosses, both of which produce a mixture of plant sexes in the seedling progenies. The development of genetic markers associated with sex expression will allow breeding programs to select the desired hermaphroditic seedlings before planting in the field, saving trellis space, and increasing efficiency. The second objective of this work is to use marker analysis of seedling progenies to better understand the inheritance of sex in muscadine.

\section{Materials and Methods}

Plant material. Muscadine vines from the progenies Ga. 12-1, Ga. 12-8, and Ga. 9-6 and their parents were grown at the University of Georgia-Tifton Campus located in Tifton, GA (lat. $33^{\circ} 53^{\prime} 7.69^{\prime \prime} \mathrm{N}$, long. $83^{\circ} 25^{\prime} 20.30^{\prime \prime} \mathrm{W}$ ). Vines from the muscadine progenies Ark. 1212 and Ark. 1214 were grown at the University of Arkansas Fruit Research Station, Clarksville, AR (lat. 35 $31^{\prime} 58^{\prime \prime} \mathrm{N}$, long. 93 $24^{\prime} 12^{\prime \prime} \mathrm{W}$ ). The parents and populations sizes for each cross are listed in Table 1. Muscadine cultivars and germplasm used in testing the association between markers and the sex trait were grown at the above two locations or at the North Carolina Department of Agriculture and Consumer Services Horticultural Crops Research Station, Castle Hayne, NC (lat. $34^{\circ} 19^{\prime} 14^{\prime \prime} \mathrm{N}$, long. $77^{\circ} 55^{\prime} 10^{\prime \prime} \mathrm{W}$ ) as listed in Supplemental Table 1. Seedling vines of the progenies were assessed for sex phenotype via visual inspection of flower morphology in Spring 2014 to Spring 2016. Sex phenotype of the cultivars and germplasm were obtained from historical records.

DNA EXtraction. Samples of young leaf tissue $\left(\approx 0.5 \mathrm{~cm}^{2}\right)$, either fresh or frozen, were placed in $2.0-\mathrm{mL}$ centrifuge tubes with screw caps. To each tube, three 3.2-mm chrome steel beads and $750 \mu \mathrm{L}$ of $2 \times$ CTAB buffer [0.1 M Tris pH8.0, $20 \mathrm{~mm}$ ethylenediaminetetraacetic acid ( $\mathrm{pH} 8.0$ ), $1.4 \mathrm{M} \mathrm{NaCl}, 2 \%$ CTAB, 0.2\% 2-mercaptoethanol] were added. Each set of tubes was arranged in a microtube rack attached to a saw blade. A reciprocating saw (JR3070CT; Makita, La Mirada, CA) was then used to grind the samples by shaking the tubes for $45 \mathrm{~s}$ on the high setting. The samples were then incubated at $65^{\circ} \mathrm{C}$ for 
Table 1. Muscadine progenies used to validate VR006 and VR009 markers for plant sex.

\begin{tabular}{|c|c|c|c|c|c|}
\hline Progeny & Seed parent & Pollen parent & Progeny sex & Seedlings (no.) $)^{z}$ & $\overline{\chi^{2} P \text { value }}$ \\
\hline \multirow[t]{5}{*}{ Ga. 12-1 } & Sugargate & Lane & $\mathrm{F}$ & 109 & \\
\hline & $\mathrm{F}$ & $\mathrm{H}$ & $\mathrm{H}$ & 87 & \\
\hline & & & Did not flower & 4 & \\
\hline & & & Total & 200 & \\
\hline & & & $\chi^{2} 1 \mathrm{~F}: 1 \mathrm{H}$ & 2.47 & 0.12 \\
\hline \multirow[t]{5}{*}{ Ga. $12-8$} & Ga. $19-37$ & Ga. 6-6-358 & $\mathrm{F}$ & 96 & \\
\hline & $\mathrm{F}$ & $\mathrm{H}$ & $\mathrm{H}$ & 103 & \\
\hline & & & Did not flower & 1 & \\
\hline & & & Total & 200 & \\
\hline & & & $\chi^{2} 1 \mathrm{~F}: 1 \mathrm{H}$ & 0.25 & 0.62 \\
\hline \multirow[t]{5}{*}{ Ga. 9-6 } & Ga. 15-19-2 & Thornhill & $\mathrm{F}$ & 95 & \\
\hline & $\mathrm{F}$ & M & M & 84 & \\
\hline & & & Did not flower & 4 & \\
\hline & & & Total & 183 & \\
\hline & & & $\chi^{2} 1 \mathrm{~F}: 1 \mathrm{M}$ & 0.68 & 0.41 \\
\hline \multirow[t]{8}{*}{ Ark. 1212} & AM-18 & AM-44 & $\mathrm{F}$ & 13 & \\
\hline & $\mathrm{H}$ & $\mathrm{H}$ & $\mathrm{H}$ & 35 & \\
\hline & & & M & 22 & \\
\hline & & & Did not flower & 5 & \\
\hline & & & Total & 75 & \\
\hline & & & $\chi^{2} 9 \mathrm{H}: 3 \mathrm{~F}: 4 \mathrm{M}$ & 1.64 & 0.44 \\
\hline & & & $\chi^{2} 2 \mathrm{H}: 1 \mathrm{~F}: 1 \mathrm{M}$ & 2.31 & 0.32 \\
\hline & & & $\chi^{2} 8 \mathrm{H}: 3 \mathrm{~F}: 4 \mathrm{M}$ & 1.15 & 0.56 \\
\hline \multirow[t]{8}{*}{ Ark. 1214} & AM-44 & AM-38 & $\mathrm{F}$ & 6 & \\
\hline & $\mathrm{H}$ & $\mathrm{H}$ & $\mathrm{H}$ & 30 & \\
\hline & & & M & 11 & \\
\hline & & & Did not flower & 8 & \\
\hline & & & Total & 55 & \\
\hline & & & $\chi^{2} 9 \mathrm{H}: 3 \mathrm{~F}: 4 \mathrm{M}$ & 1.43 & 0.49 \\
\hline & & & $\chi^{2} 2 \mathrm{H}: 1 \mathrm{~F}: 1 \mathrm{M}$ & 4.66 & 0.09 \\
\hline & & & $\chi^{2} 8 \mathrm{H}: 3 \mathrm{~F}: 4 \mathrm{M}$ & 2.74 & 0.25 \\
\hline
\end{tabular}

$\mathrm{H}=$ hermaphroditic; $\mathrm{F}=$ female; $\mathrm{M}=$ male.

${ }^{\mathrm{z}}$ Number of seedlings within the progeny of each sex.

$30 \mathrm{~min}$. To each sample, $750 \mu \mathrm{L}$ of chloroform:isoamyl alcohol (24:1) was added and mixed by vortexing. Samples were centrifuged at $16,000 g_{\mathrm{n}}$ for $10 \mathrm{~min}$ to separate the phases. Following this, $600 \mu \mathrm{L}$ of supernatant was transferred to a new $1.5-\mathrm{mL}$ centrifuge tube with flip cap, and an equal amount of isopropanol was added and mixed by inversion. The tubes were then centrifuged at $16,000 g_{\mathrm{n}}$ for $10 \mathrm{~min}$ to pellet the DNA, and the supernatant was discarded by pouring. The pellets were washed with $750 \mu \mathrm{L}$ of $70 \%$ ethanol, inverting the tubes to remove salt from the tube walls. The tubes were centrifuged again at $16,000 g_{n}$ for $5 \mathrm{~min}$, and the supernatant was discarded by pouring. The tubes were centrifuged again at $16,000 g_{n}$ for $3 \mathrm{~min}$, and the remaining ethanol was removed by pipetting with a medium tip. The samples were allowed to air dry until the remaining ethanol had evaporated and resuspended in $60 \mu \mathrm{L}$ of $50 \%$ TE with RNAse $\left(2 \mu \mathrm{L} \cdot \mathrm{mL}^{-1}\right)$ and incubated at $4{ }^{\circ} \mathrm{C}$ overnight. Before use, the samples were vortexed, centrifuged briefly, then vortexed again to dissolve the pellet and mix. DNA concentration was measured by comparison with known concentrations of $\lambda$-DNA by electrophoresis on a gel of $0.6 \%$ agarose and $0.6 \%$ synergel (Diversified Biotech, Boston, MA), run at $90 \mathrm{~V}$ for $4 \mathrm{~h}$ in $0.5 \times$ TBE buffer (Sambrook et al., 1989).

AMPLiCon Generation and SEQuence analysis. The four amplicons that were found to be linked to the sex trait in wild grape $[V$. vinifera ssp. sylvestris (Gmel.) Hegi] (VSVV006,
VSVV007, VSVV009, and VSVV010) by Picq et al. (2014) were investigated in muscadine. The forward and reverse primers (Supplemental Table 2) as described by Picq et al. (2014) were used to generate amplicons in one female, 'Thomas', one male, 'White Male', and two hermaphroditic, 'Noble' and 'Doreen', flowered muscadine cultivars. Amplifications were in a $25-\mu \mathrm{L}$ volume with $10 \mathrm{ng}$ of DNA, 1× reaction buffer, $0.2 \mathrm{~mm}$ dNTPs, 0.2 $\mu \mathrm{M}$ of each primer and 1.25 units of PrimeSTAR DNA polymerase (Takara Bio, Kusatsu, Japan). Amplification was performed in an Eppendorf scientific thermal cycler (Eppendorf Scientific, Westbury, NY) programmed for 35 amplification cycles $\left(98^{\circ} \mathrm{C} 10 \mathrm{~s}, 55^{\circ} \mathrm{C} 15\right.$ $\mathrm{s}, 68^{\circ} \mathrm{C} 40 \mathrm{~s}$ ) followed by an 8 -min extension cycle at $68{ }^{\circ} \mathrm{C}$, with maximum ramping speed between temperatures. Amplicon bands were isolated and purified using the QIAquick gel extraction kit according to instructions (Qiagen, Valencia, CA). The amplicons for each cultivar were cloned using the Zero Blunt PCR Cloning Kit according to instructions (Invitrogen, Carlsbad, CA). Amplicons were sequenced by Macrogen Corp. (Rockville, MD) and raw sequence files were imported, aligned, and trimmed using Geneious 8.0.4 software (Biomatters, Auckland, New Zealand).

VR006 AND VR009 MARKER DEVELOPMENT. Forward and reverse primers for the VR006 and VR009 markers were generated using Primer3 software (Untergasser et al., 2012) such that primers contained a minimum of $20 \%$ and an optimum of $50 \%$ guanine-cytosine bases and be 18 to $20 \mathrm{bp}$ in length. Primers were chosen from conserved regions from the four muscadine sequences in the amplicons for VSVV006 and VSVV009 such that they amplified the indel polymorphisms which distinguished the $\mathrm{F}$ from the $\mathrm{M}$ - and $\mathrm{H}$-associated alleles. Amplifications were in a $25-\mu \mathrm{L}$ volume with $10 \mathrm{ng}$ of DNA, $1 \times$ reaction buffer, $0.2 \mathrm{~mm}$ dNTPs, $0.6 \mu \mathrm{M}$ of each primer, and 1.5 units of Green Taq DNA polymerase (Genescript, Grand Cayman, Cayman Islands). Amplification was performed in a thermal cycler (Eppendorf Scientific) programmed for 35 amplification cycles $\left(94{ }^{\circ} \mathrm{C} 30 \mathrm{~s}, 57^{\circ} \mathrm{C} 1 \mathrm{~min}, 72{ }^{\circ} \mathrm{C} 1 \mathrm{~min}\right)$ followed by an 8-min extension cycle at $72{ }^{\circ} \mathrm{C}$, with maximum ramping speed between temperatures. Amplification products were resolved by electrophoresis on a gel of $0.6 \%$ agarose and $0.6 \%$ synergel (Diversified Biotech), run at $90 \mathrm{~V}$ for $4 \mathrm{~h}$ in $0.5 \times$ TBE buffer (Sambrook et al., 1989). Band sizes were estimated by comparison with a 100-bp DNA ladder.

VR006 AND VR009 MARKER ANALYSIS. Analysis of the markers in muscadine germplasm was performed by using 
a set of 122 muscadine cultivars and selections with known flower type (Supplemental Table 1). For linkage mapping, five muscadine progenies segregating for flower type were employed (Table 1). Associations among markers and flower phenotypes were tested using $\chi^{2}$ analysis.

\section{Results}

Amplicons were generated in the four muscadine cultivars for all four of the Picq et al. (2014) primer sets, VSVV006, VSVV007, VSVV009, and VSVV010. Bands of similar size to those produced in $V$. vinifera and $V$. vinifera ssp. sylvestris were generated for all primer sets except for VSVV010, which amplified poorly in the four muscadine samples. Two primer sets, VSVV006 and VSVV009, produced bands of novel sizes in muscadine germplasm. VSVV006 produced a band of the expected size of $1219 \mathrm{bp}$ in all samples; however, an additional band of $1515 \mathrm{bp}$ was also produced in the 'White Male' (M), 'Noble' (H), and 'Doreen' (H) amplifications. Sequence analysis of the two amplicons revealed the presence of a 296-bp insert in the larger amplicon (Fig. 1). The female cultivar Thomas was homozygous for the 1219-bp amplicon, which lacked the insert, whereas the male and hermaphroditic cultivars were heterozygous for the two amplicons. VSVV009 produced a 810-bp amplicon with all four DNA samples. An additional band of 916 bp was produced with the 'White Male' DNA sample. Sequence analysis revealed a 106-bp insert in the larger 'White Male' amplicon (Fig. 2). VSVV007 sequences identified single nucleotide polymorphism variation between the cultivars; however, no sex-associated polymorphism from VSVV007 was identified.

Primers based on the muscadine sequence data were designed to flank the inserts in the VSVV006 and VSVV009 amplicons to produce diagnostic markers, VR006 and VR009, for the presence of the inserts. The VR006 primer set (Supplemental Table 2) produces a 488-bp amplicon (VR006-488) when the insert is present, and a 192-bp amplicon (VR006-192)

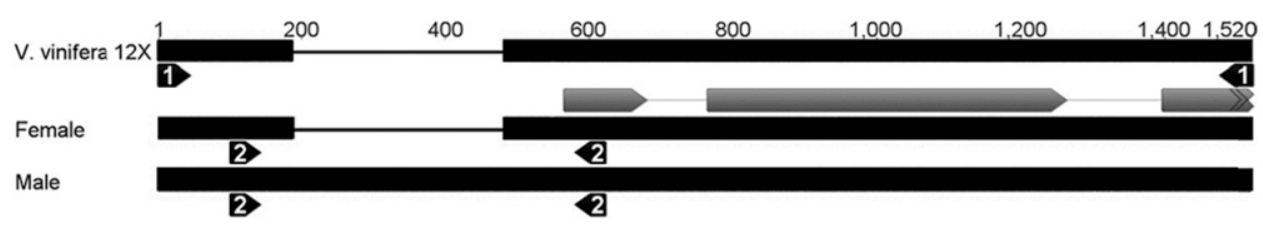

Fig. 1. Genomic structure of the VR006 amplicon in relation to grapevine trehalose-6-phosphate phosphatase gene. Vitis vinifera $12 \mathrm{X}$ sequence derived from Genbank NC_012008. Arrows labeled with a "1" indicate position of the Picq et al. (2014) VSVV006 primers. Arrows labeled with a "2" indicate VR006 forward and reverse primers which flank the male-/hermaphrodite-associated insertion. The gray boxes represent predicted exons 2-4 of LOC100852434. Deletions are shown as a thin black line.

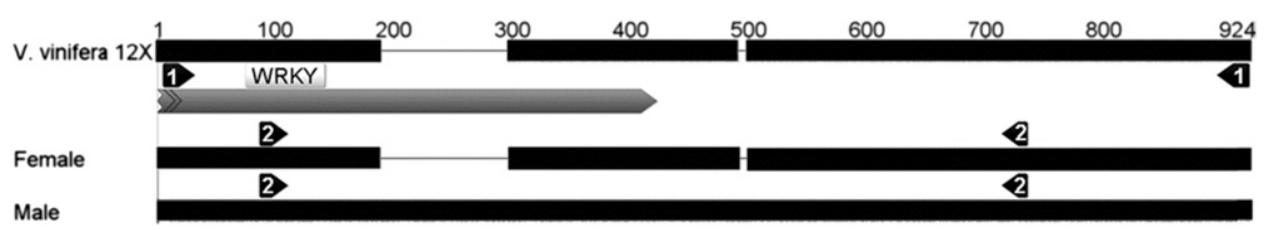

Fig. 2. Genomic structure of the VR009 amplicon in relation to grapevine. Vitis vinifera $12 \mathrm{X}$ sequence derived from Genbank NC_012008. Arrows labeled with a "1" indicate position of the Picq et al. (2014) VSVV009 primers. Arrows labeled with a " 2 " indicate VR009 forward and reverse primers which flank the male-/ hermaphrodite-associated insertion. The gray boxes represent the predicted open reading frame (GSVIVT01001286001). Deletions are shown as a thin black line. when the insert is absent. Amplification of the 488-bp amplicon was relatively poor compared with the 192-bp amplicon, especially when the 192-bp amplicon was amplified in the me reaction (Fig. 3). The VR009 primer set (Supplemental Theduces a 646-bp amplicon (VR009-646) when the insert is present, and a 540-bp amplicon (VR009-540) when the insert is absent. Size differences for each marker are readily (Fig. 3) Termplasm of known sex (Supplemental Table 1). This germplasm consisted of modern cultivars, old cultivars, breeding program selections and is representative of the germplasm consisted of 57 females, 61 hermaphrodites, and of hermaphrodites made them obsolete and most males were subsequently discarded.

VR006 exhibited a polymorphism between females and males or hermaphrodites. All 57 females were homozygous for the VR006-192 allele (Table 2). All males were heterozygous displaying both the 192- and 488-bp alleles. Most, 57 of , of the hermaphrodites were heterozygous, whereas four indicate a dominant relationship with males and hermaphrodites having at least one copy of the 488-bp allele, whereas males were homozygous recessive for the 192-bp allele.

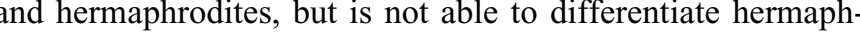
rodites from males. The four clones homozygous for the 488-bp allele were all recent breeding program selections that were females (Table 2). The four males were heterozygous for the 540- and 646-bp alleles. The hermaphrodites had one of three different genotypes. Four clones were homozygous for the 646bp allele. These four clones are the same four clones that were homozygous for VR006-488. Out of the remaining hermaphrodites, 37 were heterozygous and 20 were homozygous for the 540-bp allele. Thus, VR009 is not useful in differentiating hermaphrodites from females in unrelated germplasm. Examination of the pedigrees of the 20 hermaphrodites homozygous for VR009-540 (Supplemental Table 1) revealed a strong association among these cultivars. Twelve of the 20 clones can have their pollen parent pedigree traced back to the cultivar Tarheel (Supplemental Fig. 1). Two other cultivars, Dixie and Doreen, have a parent-sibling relationship, and the other five clones have no known relationship, but the pedigrees in several cases are incomplete.

Six mapping populations were chosen to test the linkage between the markers and sex phenotype. Two progenies, Ga. 12-1 and Ga. 12-8, were derived from $\mathrm{F} \times \mathrm{H}$ crosses. Both progenies consisted of 200 seedlings that segregated in 
a 1:1 F:H ratio (Table 1). Ga. 9-6 consisted of 183 seedlings from a cross of a female muscadine and a male $V$. munsoniana parent, and segregated in a 1:1 F:M ratio (Table 1). Progenies Ark. 1212 and Ark. 1214 were produced by emasculating a hermaphrodite and pollinating it with a different hermaphrodite. Both of these populations could fit various segregation ratios including a 9:3:4, 2:1:1, or 8:3:4 H:F:M ratio (Table 1).

In progenies Ga. 12-1 and Ga. 12-8, all females were homozygous for marker VR006-192 and all hermaphrodites were heterozygous VR006-192 + VR006-488 (Table 3). Among the seedlings that did not flower, three were homozygous VR006-192 and two were heterozygous. In progeny Ga. 9-6, all females were homozygous for marker VR006-192 and all males were heterozygous (Table 3 ). In each of these progenies, no recombination was detected between the VR006 marker and the flower sex locus. In Ark. 1212 and Ark. 1214, all seedlings that were homozygous VR006-192 were female and all seedlings that were homozygous VR006-488 were hermaphroditic. Seedlings that were heterozygous for the VR006 marker segregated in a 1:1 H:M ratio in Ark. $1212\left(\chi^{2}=0.012, P=0.99\right)$ and in Ark. $1214\left(\chi^{2}=0.36, P=0.84\right)$. Thus, while the flower sex of both homozygotes could be predicted, the flower sex of heterozygous individuals could not be predicted based on VR006 genotype. Of the five vines that did not flower in Ark. 1212, three were homozygous VR006-192, one was homozygous VR006-488 and one was heterozygous. Of the eight vines that did not flower in Ark. 1214, four were homozygous VR006-192, one was homozygous VR006-488, and three were heterozygous.

Marker genotypes of VR009 mirrored exactly those of VR006, with the allele containing the insert, VR009-646, again associated with hermaphrodites and males (Table 4). Crossover events between VR006 and VR009 would be evidenced by an individual being homozygous for one marker and heterozygous for the other. No progeny displayed this marker pattern (Tables 3 and 4), indicating that the two markers are very tightly linked.

\section{Discussion}

The genus Vitis consists of about 60 species, all of which are dioecious in nature. However, the domesticated grape vine, $V$. vinifera, consists primarily of hermaphrodites. The origin and selection of hermaphrodism occurred early in the domestication of this species and the process of this event remains unknown. The molecular basis of the sex trait in Vitis remains poorly understood. Mapping studies indicate that sex expression in Euvitis appears to be under control of a single genomic region located on chromosome 2, close to SSR

Table 3. Association between marker VR006 and the sex trait in muscadine progenies.

\begin{tabular}{|c|c|c|c|c|c|c|}
\hline Progeny & $\begin{array}{l}\text { Female parent } \\
\text { allele sizes (bp) }\end{array}$ & $\begin{array}{l}\text { Pollen parent } \\
\text { allele sizes (bp) }\end{array}$ & $\begin{array}{c}\text { Progeny } \\
\text { allele sizes (bp) }\end{array}$ & $\begin{array}{l}\text { Hermaphrodites } \\
\text { (no.) }\end{array}$ & $\begin{array}{c}\text { Females } \\
\text { (no.) }\end{array}$ & $\begin{array}{c}\text { Males } \\
\text { (no.) }\end{array}$ \\
\hline \multirow[t]{2}{*}{ Ga. 12-1 } & Sugar Gate (F) & Lane $(\mathrm{H})$ & $488+192$ & 87 & 0 & 0 \\
\hline & $192+192$ & $488+192$ & $192+192$ & 0 & 109 & 0 \\
\hline & $192+192$ & $488+192$ & $192+192$ & 0 & 96 & 0 \\
\hline \multirow[t]{2}{*}{ Ga. 9-6 } & Ga. 15-19-2 (F) & Thornhill (M) & $488+192$ & 0 & 0 & 84 \\
\hline & $192+192$ & $488+192$ & $192+192$ & 0 & 95 & 0 \\
\hline & $488+192$ & $488+192$ & $488+192$ & 21 & 0 & 22 \\
\hline & & & $192+192$ & 0 & 13 & 0 \\
\hline \multirow[t]{3}{*}{ Ark. 1214} & AM-44 (H) & AM-38 (H) & $488+488$ & 16 & 0 & 0 \\
\hline & $488+192$ & $488+192$ & $488+192$ & 14 & 0 & 11 \\
\hline & & & $192+192$ & 0 & 6 & 0 \\
\hline
\end{tabular}

$\mathrm{H}=$ hermaphroditic, $\mathrm{F}=$ female, $\mathrm{M}=$ male. 
Table 4. Association between marker VR009 and the sex trait in muscadine progenies.

\begin{tabular}{|c|c|c|c|c|c|c|}
\hline Progeny & $\begin{array}{l}\text { Female parent } \\
\text { allele sizes (bp) }\end{array}$ & $\begin{array}{l}\text { Pollen parent } \\
\text { allele sizes }(\mathrm{bp})\end{array}$ & $\begin{array}{c}\text { Allele } \\
\text { sizes (bp) }\end{array}$ & $\begin{array}{l}\text { Hermaphrodites } \\
\text { (no.) }\end{array}$ & $\begin{array}{l}\text { Females } \\
\text { (no.) }\end{array}$ & $\begin{array}{c}\text { Males } \\
\text { (no.) }\end{array}$ \\
\hline \multirow[t]{2}{*}{ Ga. $12-1$} & Sugar Gate (F) & Lane $(\mathrm{H})$ & $646+540$ & 87 & 0 & 0 \\
\hline & $540+540$ & $646+540$ & $540+540$ & 0 & 109 & 0 \\
\hline & $540+540$ & $646+540$ & $540+540$ & 0 & 96 & 0 \\
\hline \multirow[t]{2}{*}{ Ga. 9-6 } & Ga. 15-19-2 (F) & Thornhill (M) & $646+540$ & 0 & 0 & 84 \\
\hline & $540+540$ & $646+540$ & $540+540$ & 0 & 95 & 0 \\
\hline & $646+540$ & $646+540$ & $646+540$ & 21 & 0 & 22 \\
\hline & & & $540+540$ & 0 & 13 & 0 \\
\hline \multirow[t]{3}{*}{ Ark. 1214} & AM-44 (H) & AM-38 (H) & $646+646$ & 16 & 0 & 0 \\
\hline & $646+540$ & $646+540$ & $646+540$ & 14 & 0 & 11 \\
\hline & & & $540+540$ & 0 & 6 & 0 \\
\hline
\end{tabular}

$\mathrm{H}=$ hermaphroditic, $\mathrm{F}=$ female, $\mathrm{M}=$ male.

marker VVIB23 (Dalbó et al., 2000; Lowe and Walker, 2006; Marguerit et al., 2009; Riaz et al., 2006). Candidate genes in the sex-linked region have recently been investigated and described (Fechter et al., 2012; Picq et al., 2014).

Picq et al. (2014) partially sequenced 11 genes in the region of the sex locus in $V$. vinifera ssp. sylvestris. Four of these amplicons showed polymorphisms significantly associated to the sex trait. In $V$. vinifera ssp. sylvestris, $100 \%$ of the females were homozygous for the most frequent allele, and $100 \%$ of the males were heterozygous. These amplicons were determined to be part of a small XY sex-determining region with XY males and XX females. In hermaphroditic-domesticated $V$. vinifera, the majority of alleles were also heterozygous, with the hermaphroditic alleles deriving from the male alleles of wild grapevines. Our results in $V$. rotundifolia show a similar pattern in that the male and hermaphrodite alleles both possess insertions not found in the female allele. VR006 amplifies the first intron through the fourth exon of a TPP gene (Fig. 1). Expression of grape SISTER OF RA3 (VvSRA), also a TPP gene, is negatively related to pedicel development on the lateral branch of grape inflorescences, thereby decreasing berry number (Ishiai et al., 2015). Male and hermaphroditic muscadine inflorescences generally produce more flowers than do female inflorescences (Biasi and Conner, 2016; Detjen, 1917). Expression studies of the VR006 gene may help in determining if this gene could play a role in sex determination in muscadine.

The VR009 amplicon has sequence similarity to a WRKY transcription factor 21. The WRKY transcription factor family is one of the largest gene families involved in plant development and stress responses (Xu et al., 2016). However, the presence of hermaphrodites homozygous for the femaleassociated allele VR009-192 precludes a direct relationship between VR009 and sex phenotype.

Compared with $V$. vinifera, the domestication of $V$. rotundifolia is relatively recent, and the origin of hermaphrodism is well documented in the scientific literature (Dearing, 1917, 1948). Despite their distinct origin, morphologically the three flower types in $V$. rotundifolia appear similar to those of $V$. vinifera (Detjen, 1917; Oberle, 1938). The proposed model for inheritance of flower type of Oberle (1938) is intriguing because a crossover event between the linked genes would lead to the formation of hermaphrodites. The discovery of three wild hermaphrodites (Detjen, 1917) and the production of four separate hermaphrodites from relatively modest population sizes (Dearing 1948), and the similar appearance of hermaphrodism in separate species suggests that crossover events between gene regions, rather than mutation, may be the more likely source of hermaphrodism in $V$. rotundifolia.

Genetic ratios obtained in the $\mathrm{F} \times \mathrm{M}$ and $\mathrm{F} \times \mathrm{H}$ crosses in this study produced the 1:1 ratios that were obtained with similar crosses in previous studies (Dearing, 1917; Detjen, 1917; Loomis, 1948). In contrast to the results of Loomis (1948) and Dearing (1917), no male plants were seen in the two $\mathrm{F} \times \mathrm{H}$ crosses. The small number of male plants produced in the $\mathrm{F} \times \mathrm{H}$ crosses of Dearing (1917) and Loomis (1948) were attributed to pollen contamination or seed handling errors. The lack of male seedlings in this work may be attributed to better breeding practices or to a general lack of male plants in current muscadine germplasm so that any outcrosses would also be female or hermaphroditic.

Selfs of hermaphroditic muscadines have produced two types of progenies. Selfs of hermaphrodites descended from $\mathrm{H} 1$ segregated in a 3:1 H:F ratio, whereas those descended from H2 or 'Hope' segregated in a 9:3:4 H:F:M ratio (Detjen, 1917; Loomis et al., 1954). The presence of males in these progenies is not predicted by the genetic model proposed by Oberle (1938), and their genetic origin is unexplained. Both the Ark. 1212 and the Ark. 1214 progeny produced a large number of male plants. $\chi^{2}$ analysis of the progeny ratios indicate that both progenies fit the 9:3:4 H:F:M ratio produced by Loomis et al. (1954) for selfs of H2-descended hermaphrodites (Table 1). Examination of the pedigrees of AM-18 and AM-38, two of the three parents used in crosses Ark. 1212 and Ark. 1214, show that they can be traced through the male line to H1 (Supplemental Fig. 2). However, this pedigree is questionable because AM-18, AM-38, 'Tara', and 'Triumph' all have one copy of the VR009-646 allele, whereas 'Tarheel' is homozygous VR009540 (Supplemental Table 1). If the male line was descended from 'Tarheel' as the pedigree indicates, these clones could not have inherited the VR009-646 allele. Thus, it seems likely that an outcross event occurred somewhere in the pedigree between 'Tarheel' and 'Triumph'. Since the selections Ga. 1 and Ga. 2949 no longer exist, it is impossible to test this conclusion further. The male line pedigree of AM-44, the third parent in these crosses, involves two instances of open pollination and is thus unable to be traced unambiguously to its hermaphroditic source (Supplemental Fig. 2). 
The marker pattern of the Ark. 1212 and Ark. 1214 progenies were examined to provide information on the inheritance of flower type. Both progenies segregated in a 1:2:1 homozygous dominant:heterozygous:homozygous recessive ratio for both markers (Ark. $1212 \chi^{2}=3.7, P=0.16$; Ark. $\left.1214 \chi^{2}=4.4, P=0.11\right)$. In both crosses, all progeny that were homozygous for VR006-488 and VR009-646 were hermaphroditic, and all progeny that were homozygous for VR006-192 and VR009-488 were female (Tables 3 and 4). The seedlings that were heterozygous for each marker segregated in a 1:1 ratio of $\mathrm{M}: \mathrm{H}$. This result predicts a phenotypic ratio of $2: 1: 1 \mathrm{H}: \mathrm{F}: \mathrm{M}$ rather than the 9:3:4 H:F:M ratio suggested by Loomis et al. (1954). $\chi^{2}$ analysis indicates that the Ark. 1212 and Ark. 1214 progenies can fit a $2: 1: 1 \mathrm{H}: \mathrm{F}: \mathrm{M}$ ratio, but the fit is not as good as the 9:3:4 H:F:M ratio (Table 1). Across several selfed progenies, Loomis et al. (1954) produced a ratio of 535:187:259 H:F: M seedlings. The fit of these progenies for a 9:3:4 H:F:M ratio is very $\operatorname{good}\left(\chi^{2}=1.33, P=0.51\right)$, but the data does not fit a $2: 1: 1$ $\mathrm{H}: \mathrm{F}: \mathrm{M}$ ratio $\left(\chi^{2}=18.6, P<0.001\right)$. Examination of the observed and expected numbers for the $2: 1: 1 \mathrm{H}: \mathrm{F}: \mathrm{M}$ ratio shows a consistent lack of $\approx 6 \%$, or $1 / 16$, of female seedlings. A second unlinked factor, heterozygous in both parents, which was lethal when homozygous in the VR006-192/VR009-488 homozygote would produce an 8:3:4 H:F:M ratio. Ark. 1212, Ark. 1214 (Table 1), and the Loomis et al. (1954) progenies $\left(\chi^{2}=\right.$ $4.85, P=0.09)$ could all fit the $8: 3: 4 \mathrm{H}: \mathrm{F}: \mathrm{M}$ ratio, but the fit is not as good as the 9:3:4 H:F:M ratio.

As Loomis et al. (1954) stated, more extensive data are needed to understand the inheritance of flower type in muscadine. Ideally, the profiles of the VR006 and VR009 markers would be included in the design and analysis of new genetic tests. Particular crosses of interest include a) intercrosses of H1 and $\mathrm{H} 2$ descended hermaphroditic parents, b) $\mathrm{H} 2$ intercross derived females $\times \mathrm{H} 2$ descended hermaphrodites, c) $\mathrm{H} 2$ intercross derived males $\times$ females, d) $\mathrm{H} 2$ intercross derived males $\times$ hermaphrodites, and e) VR006-488/VR009-646 homozygotes $\times$ females.

Despite the ambiguity of the inheritance of flower type, the markers developed in this project have great utility for breeding programs. The VR markers developed here fulfill the requirements laid out by Heikrujam et al. (2015) for ideal PCR-based markers. The markers produce a band in all individuals which can serve as a positive control and an additional diagnostic band that defines the sex of the individual ( $\mathrm{F}$ or $\mathrm{H} / \mathrm{M}$ ). In addition, the size differences between the alleles are large enough that they can easily be identified on a standard agarose gel. Most breeding programs are focusing on releasing hermaphroditic cultivars due to their associated yield gain and lack of a need for a pollinizer (Conner, 2014). Crosses used to develop new cultivars are normally $\mathrm{F} \times \mathrm{H}$ or $\mathrm{H} \times \mathrm{H}$. The percentage of hermaphroditic progeny produced in these crosses would vary from $50 \%$ to $75 \%$ depending on the type of cross and the source of hermaphrodism. The utility of the marker will vary depending on the cross. In $\mathrm{F} \times \mathrm{H}$ crosses (and $\mathrm{F} \times \mathrm{M}$ crosses though they are seldom used), the VR006 marker will allow determination of the sex of all the progeny. In crosses between hermaphrodites, the VR006 marker will allow females to be identified, but will not be able to discriminate between males and hermaphrodites.

Overall, VR006 has greater utility than VR009 because all male and hermpaphroditic clones have at least one copy of the VR006-488 allele. However, if the parents have been screened and are known to be segregating for VR009, this marker will work as well as VR006 in screening breeding progenies. In addition to being useful in identifying and removing females from segregating populations, these markers are also able to identify progeny from $\mathrm{H} \times \mathrm{H}$ crosses which are homozygous for the hermaphroditic allele. It is expected that when these clones are used as parents in crosses to females they will produce progeny that are entirely hermaphroditic, and would thus be superior parents. Although this cross has not yet been carried out, Loomis and Williams (1957) previously identified a hermaphrodite derived from a self of the hermaphroditic cultivar Dearing, which appeared to be homozygous for the hermaphroditic character and produced entirely hermaphroditic progenies in crosses to female cultivars. We expect these markers to be used by breeding programs both in planning crosses and selecting seedlings to increase efficiency.

\section{Literature Cited}

Antcliff, A.J. 1980. Inheritance of sex in Vitis. Ann. Amelior. Plant. 30:113-122.

Battilana, J., S. Lorenzi, F.M. Moreira, P. Moreno-Sanz, O. Failla, F. Emanuelli, and M. Stella Grando. 2013. Linkage mapping and molecular diversity at the flower sex locus in wild and cultivated grapevine reveal a prominent SSR haplotype in hermaphroditic plants. Mol. Biotechnol. 54:1031-1037.

Biasi, L. and P. Conner. 2016. Reproductive traits of hermaphroditic muscadine cultivars. HortScience 51:255-261.

Carbonneau, A. 1983. Male and female sterility in the genus Vitis, II: Consequences for genetics and breeding. Agronomie 3:645-649.

Conner, P. 2014. Characteristics of promising muscadine grape (Vitis rotundifolia Michx.) selections from the University of Georgia (U.S.A) breeding program. Acta Hort. 1046:303-307.

Dalbó, M.A., G.N. Ye, N.F. Weeden, H. Steinkellner, K.M. Sefc, and B.I. Reisch. 2000. A gene controlling sex in grapevines placed on a molecular marker-based genetic map. Genome 43:333-340.

Dearing, C. 1917. The production of self-fertile muscadine grapes. Proc. Amer. Soc. Hort. Sci. 14:30-34.

Dearing, C. 1948. New muscadine grapes. U.S. Dept. Agr. Circ. 769. Detjen, L. 1917. Inheritance of sex in Vitis rotundifolia. North Carolina Agr. Expt. Sta. Tech. Bul. 17.

Fechter, I., L. Hausmann, M. Daum, T.R. Sörensen, P. Viehöver, B. Weisshaar, and R. Töpfer. 2012. Candidate genes within a $143 \mathrm{~kb}$ region of the flower sex locus in Vitis. Mol. Genet. Genomics 287:247-259.

Heikrujam, M., K. Sharma, M. Prasad, and V. Agrawal. 2015. Review on different mechanisms of sex determination and sex-linked molecular markers in dioecious crops: A current update. Euphytica 201:161-194.

Ishiai, S., Y. Nakajima, S. Enoki, and S. Suzuki. 2015. Grape SISTER OF RAMOSA3 is a negative regulator of pedicel development of grape inflorescence. Plant Cell Tissue Organ Cult. 124:217-225.

Levadoux, L. 1946. Study of the flower and sexuality in grapes. Annales de l'École nationale d'agriculture de Montpellier 27:1-89.

Loomis, N.H. 1948. A note on the inheritance of flower type in muscadine grapes. Proc. Amer. Soc. Hort. Sci. 52:276-278.

Loomis, N.H. and C.F. Williams. 1957. A new genetic flower type of the muscadine grape. J. Hered. 48:294-304.

Loomis, N.H., C.F. Williams, and M.M. Murphy. 1954. Inheritance of flower types in muscadine grapes. Proc. Amer. Soc. Hort. Sci. 64:279-283.

Lowe, K.M. and M.A. Walker. 2006. Genetic map of the interspecific grape rootstock cross Ramsey (Vitis champanii) $\times$ Riparia Gloire (Vitis riparia). Theor. Appl. Genet. 112:1582-1592.

Marguerit, E., C. Boury, A. Manicki, M. Donnart, G. Butterlin, A. Némorin, S. Wiedemann-Merdinoglu, D. Merdinoglu, N. Ollat, and S. Decroocq. 2009. Genetic dissection of sex determinism, inflorescence 
morphology and downy mildew resistance in grapevine. Theor. Appl. Genet. 118:1261-1278.

Oberle, G.D. 1938. A genetic study of variations in floral morphology and function in cultivated forms of Vitis. New York State Agr. Expt. Sta. Tech. Bul. 250:416-420.

Picq, S., S. Santoni, T. Lacombe, M. Latreille, A. Weber, M. Ardisson, S. Ivorra, D. Maghradze, R. Arroyo-Garcia, P. Chatelet, P. This, J. Terral, and R. Bacilieri. 2014. A small XY chromosomal region explains sex determination in wild dioecious $V$. vinifera and the reversal to hermaphroditism in domesticated grapevines. BMC Plant Biol. 14:1-32.

Reimer, F.C. and L.R. Detjen. 1910. Self-sterility of the Scuppernong and other muscadine grapes. North Carolina Agr. Expt. Sta. Bul. 201.
Riaz, S., A.F. Krivanek, K. Xu, and M.A. Walker. 2006. Refined mapping of the pierce's disease resistance locus, PdR1 and sex on an extended map of Vitis rupestris $\times$ V.arizonica. Theor. Appl. Genet. 113:1317-1329.

Sambrook, J., E. Fritsch, and T. Maniatis. 1989. Molecular cloning. 2nd ed., Vol. 1. Cold Spring Harbor Lab. Press, Cold Spring Harbor, NY.

Untergasser, A., I. Cutcutache, T. Koressaar, J. Ye, B. Faircloth, M. Remm, and S.G. Rozen. 2012. Primer3-new capabilities and interfaces. Nucleic Acids Res. 40:e115.

Xu, H.J., K.A. Watanabe, L.Y. Zhang, and Q.X.J. Shen. 2016. WRKY transcription factor genes in wild rice Oryza nivara. DNA Res. 23:311-323. 


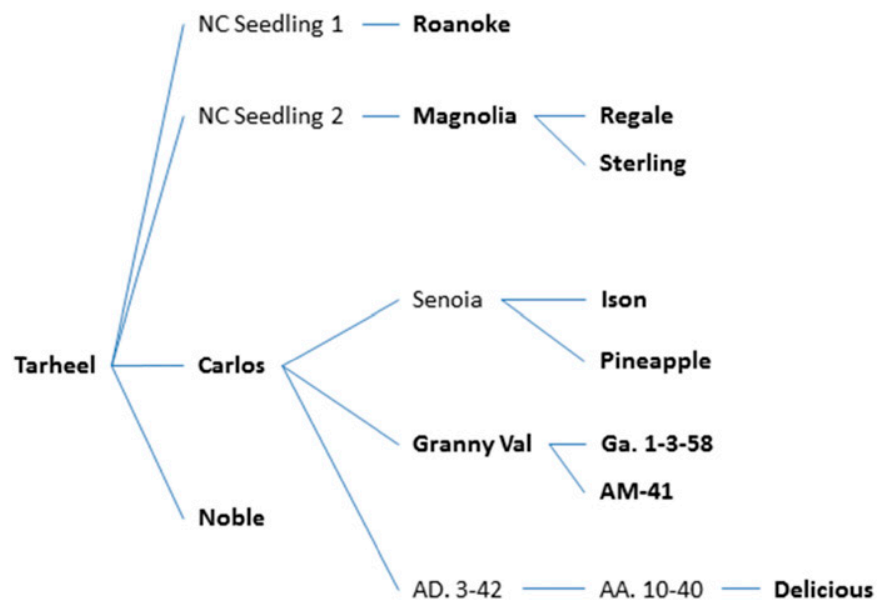

Supplemental Fig. 1. Male line pedigree of 13 hermaphroditic muscadines homozygous for marker VR009-540 (shown in bold). The five remaining clones were not available for testing and their genotype is unknown. 

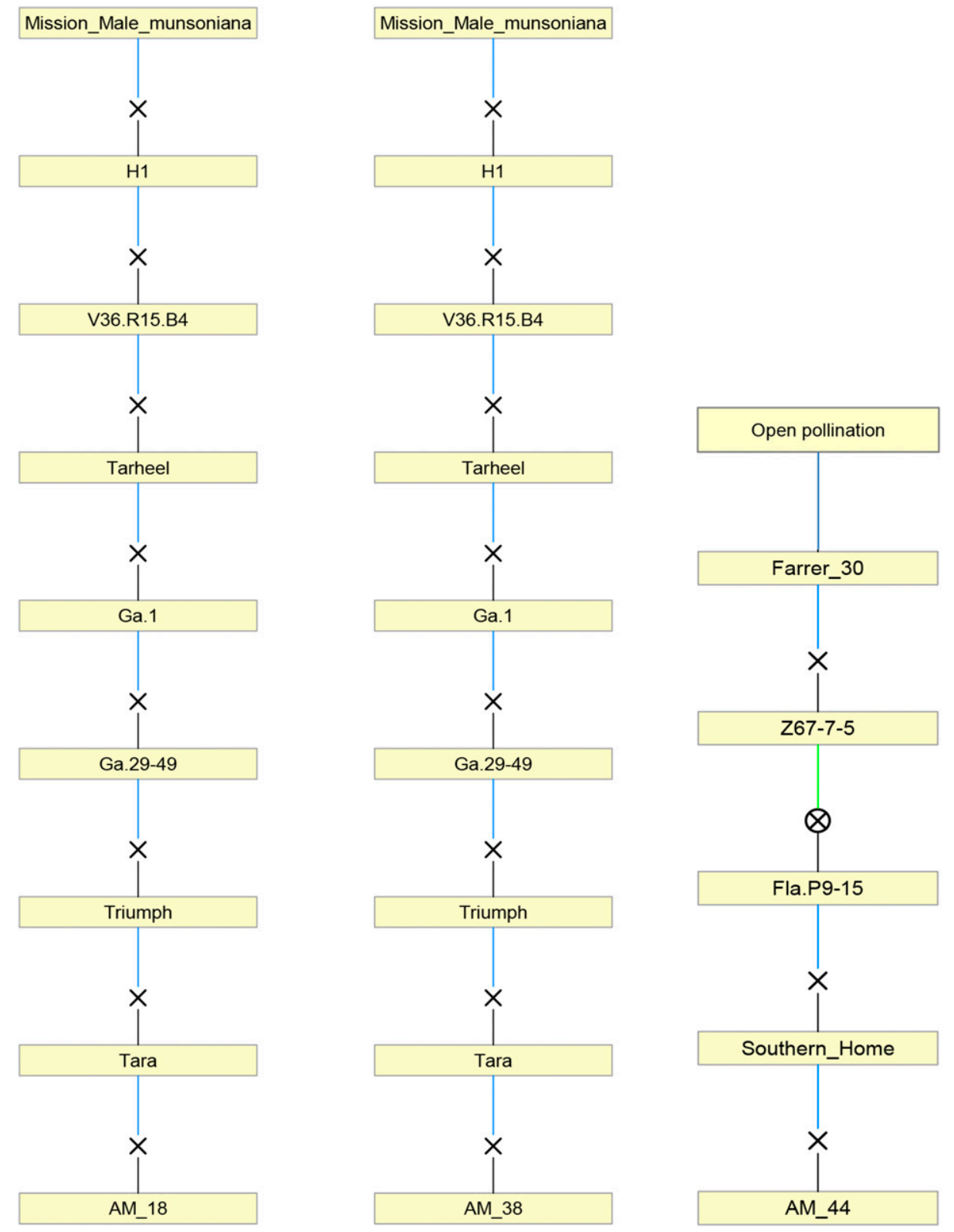

Supplemental Fig. 2. Male line pedigree of muscadine parents AM-18, AM-38, and AM-44.

Supplemental Table 1. Vine location, vine sex, and VR006 and VR009 marker profiles of 122 muscadine clones.

\begin{tabular}{|c|c|c|c|c|c|c|}
\hline \multirow[b]{2}{*}{ Clone $^{z}$} & \multirow[b]{2}{*}{ Vine sex } & \multirow[b]{2}{*}{ Vine location } & \multicolumn{2}{|c|}{ VR006 } & \multicolumn{2}{|c|}{ VR009 } \\
\hline & & & $488 \mathrm{bp}$ & $192 \mathrm{bp}$ & $646 \mathrm{bp}$ & $540 \mathrm{bp}$ \\
\hline Alachua & Perfect & Tifton, GA & $\mathrm{X}^{\mathrm{y}}$ & $\mathrm{X}$ & $\mathrm{X}$ & $\mathrm{X}$ \\
\hline AM-18 & Perfect & Clarksville, AR & $\mathrm{X}$ & $\mathrm{X}$ & $\mathrm{X}$ & $\mathrm{X}$ \\
\hline AM-26 & Perfect & Clarksville, AR & $\mathrm{X}$ & $\mathrm{X}$ & $\mathrm{X}$ & $\mathrm{X}$ \\
\hline AM-3 & Perfect & Clarksville, AR & $X$ & $\mathrm{X}$ & $\mathrm{X}$ & $\mathrm{X}$ \\
\hline AM-43 & Perfect & Clarksville, AR & $\mathrm{X}$ & $\mathrm{X}$ & $\mathrm{X}$ & $\mathrm{X}$ \\
\hline AM-44 & Perfect & Clarksville, AR & $X$ & $\mathrm{X}$ & $\mathrm{X}$ & $\mathrm{X}$ \\
\hline AM-45 & Perfect & Clarksville, AR & $\mathrm{X}$ & $\mathrm{X}$ & $\mathrm{X}$ & $X$ \\
\hline AM-53 & Perfect & Clarksville, AR & $X$ & & $\mathrm{X}$ & \\
\hline AM-56 & Perfect & Clarksville, AR & $\mathrm{X}$ & & $\mathrm{X}$ & \\
\hline
\end{tabular}


Supplemental Table 1. Continued.

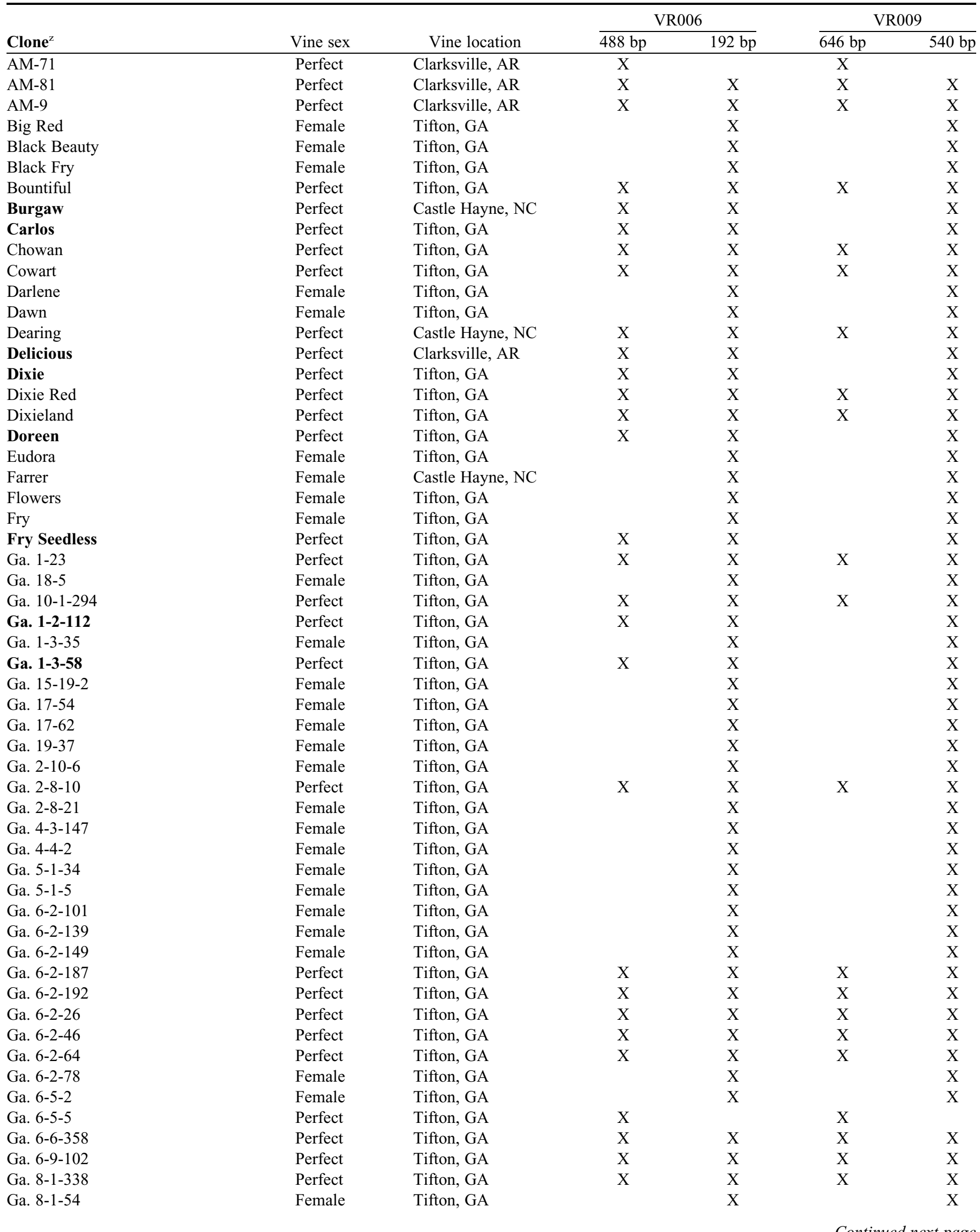


Supplemental Table 1. Continued.

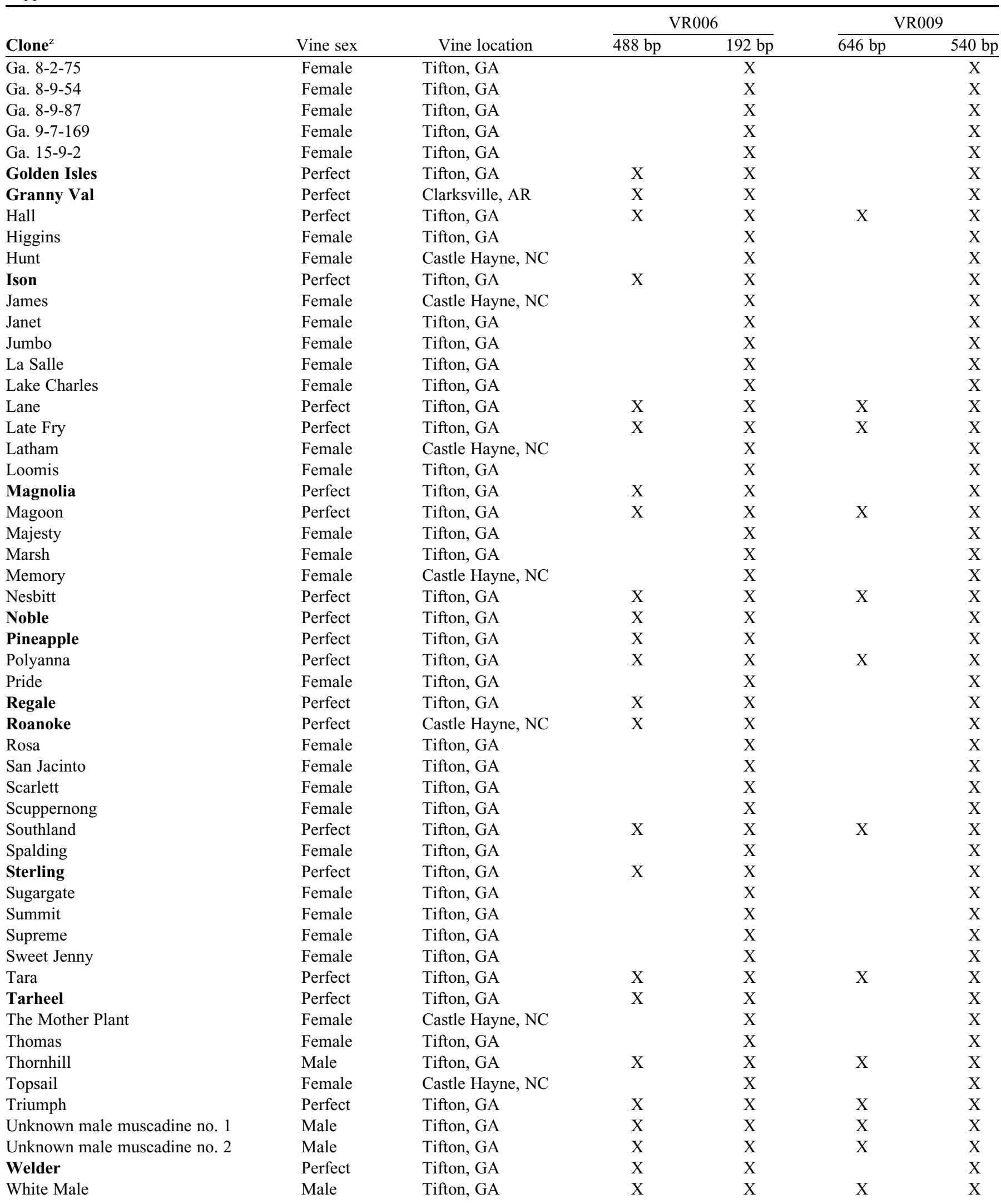

${ }^{\mathrm{z}}$ Clones in bold font have different profiles for markers VR006 and VR009.

${ }^{\mathrm{y}} \mathrm{X}$ denotes presence of allele. 
Supplemental Table 2. Primers sequences used to generate initial amplicons and VR006 and VR009 markers in muscadine grape.

\begin{tabular}{lll}
\hline Amplicon & \multicolumn{1}{c}{ Forward primer } & \multicolumn{1}{c}{ Reverse primer } \\
\hline VSVV006 $^{z}$ & TGGATTAGCTTGGCGAATAAA & CACGGTCAGGATCATCAACA \\
VSVV007z & CTCTCCGTTTTCCATTTCCA & TCCTCAACCCCAGTGAACTC \\
VSVV009 & AGGAAACTGAGGATAAAGAGATCGT & GGATAGACAGCGAGTTAGCACTTAG \\
VSVV010 & AGTGCTCACTTTTCCTTGTGAA & CATGAATCAGCAGTGCATTT \\
VR006 & TCGACCTGATATTTGGATTTTG & TGATAGACAGCTTTCCAATTCTGA \\
VR009 & GGAGGAAATATGGGCAAAAGCC & GAGCTGCCCCCTCTTGTTAG
\end{tabular}

${ }^{\mathrm{z} P r i m e r s}$ for VSVV006, VSVV007, VSVV009, and VSVV010 from Picq et al. (2014). 